\title{
Calibration of Trust in Automated Driving: a Matter of Initial LeVel of Trust and Automated Driving Style?
}

\section{J. B. Manchon ${ }^{1,2}$, Mercedes Bueno', and Jordan Navarro ${ }^{2,3}$}

${ }^{1}$ VEDECOM Institute, Versailles, France

${ }^{2}$ Laboratoire d'Etude des Mécanismes Cognitifs (EA 3082), University Lyon 2, Bron, France

${ }^{3}$ Institut Universitaire de France, Paris, France

Correspondence address: J. B. Manchon, VEDECOM Institute, 23 bis Allée des Marronniers, 78000 Versailles, France; e-mail: jb.manchon@vedecom.fr.

\section{ACKNOWLEDGMENTS}

Images : Some graphics include pictograms by Freepik from flaticon.com and from iconmonstr.com.

The authors declare that they have no known competing financial interests or personal relationships that could have appeared to influence the work reported in this paper. 


\section{ABSTRACT}

Automated driving is becoming a reality, such technology raises new concerns about human-machine interaction on-road. Sixty-one drivers participated in an experiment aiming to better understand the influence of initial level of trust (Trustful vs Distrustful) on drivers' behaviors and trust calibration during simulated Highly Automated Driving (HAD). The automated driving style was manipulated as positive (smooth) or negative (abrupt) to investigate human-machine early interactions. Trust was assessed over time through questionnaires. Drivers' visual behaviors and take-over performances during an unplanned take-over request were also investigated. Results showed an increase of trust in automation over time, for both Trustful and Distrustful drivers regardless the automated driving style. Trust was also found to fluctuate over time depending on the specific events handled by the automated vehicle. Take-over performances were not influenced by the initial level of trust nor automated driving style.

Keywords: trust in automation, automated driving, driver's behaviors, automation driving style, trust calibration 


\section{INTRODUCTION}

Automated Driving (AD) seems to be one of the disruptive technologies that promise a revolution in mobility for the upcoming decades. Yet the automation process leading to self-driving cars fleet is progressive, and drivers are facing a transition from manual driving to increasingly more automated features for these evolutions to come (T. Zhang et al., 2019).

Trust in Automation (TiA) has been shown to strongly influence automation acceptance (e.g., Beggiato \& Krems, 2013; Ghazizadeh et al., 2012; Navarro, 2019). Furthermore, human's level of TiA has been linked with automation use (Lee \& Moray, 1994), misuse or disuse (Parasuraman \& Riley, 1997). As a matter of road safety, it appears important to understand the impact of trust on $A D$ use, to provide recommendations to future vehicles design. According to Hoff and Bashir (2015), TiA is built on three layers: the dispositional trust, related to the operator inherent characteristics; the situational trust, which vary depending on the environment; and the learned trust, influenced by the (i) automation specificities, (ii) operator previous experiences with automation (initial learned trust), and (iii) operator-automation dynamic interactions (dynamic learned trust). The initial learned trust relies on the operator's beliefs and knowledges about a considered automation. This trust layer has a strong influence on the intention to use the system and the early interactions. The factors related to the dynamic learned trust then becomes more influent during the interaction, when the operator gains knowledge and experience with the automation. As automated driving technologies are progressively becoming available, it is of particular interest to investigate the specificities of drivers' Trust in Automated 
Driving (TiAD), in order to understand the cognitive processes underlying automated vehicles adoption (Manchon et al., 2020).

TiAD has been studied during the last years (e.g., Beller et al., 2013; Kraus, Scholz, Messner, et al., 2020; Payre et al., 2016; Schwarz et al., 2019), but little is known about its early calibration (Hergeth et al., 2016; Körber, Baseler, et al., 2018) and its evolution over time (Hartwich, Witzlack, et al., 2018; Walker et al., 2018). Following Hoff and Bashir's theoretical framework (2015), this study focus on the initial and dynamic learned trust. It aims to better understand the early calibration of this dynamic learned trust over time, during AD. Another related objective is to better apprehend the influence of the initial learned trust on this dynamic layer.

Initial learned trust is defined here as driver's initial level of TiAD before any interaction with an automated driving system. This initial level of trust is influenced by drivers' mental model (Beggiato et al., 2015), expectations (Hergeth et al., 2017; Körber, Baseler, et al., 2018), prior experiences with other systems (Schwarz et al., 2019; Walker et al., 2019), and perceived understanding (Khastgir et al., 2018). Based on those elements, drivers can be classified as Trustful or Distrustful according to their initial level of trust (Manchon et al., submitted). Dynamic learned trust is expected to fluctuate during actual interaction with automation (Bueno et al., 2016; Feldhütter et al., 2016; Gold et al., 2015), depending on AD's features (Miller et al., 2016; Payre et al., 2017), performance (Abe et al., 2018; Morris et al., 2017), and type of feedback provided (Häuslschmid et al., 2017; Koo et al., 2015; Lu et al., 2019; Wintersberger et al., 2017). These factors are expected to induce periodic trust recalibrations. Previous studies showed that automation driving style was 
able to influence dynamic trust calibration (e.g., Hartwich et al., 2018). In an experiment, Morris et al. (2017) determined that a risky automated drive (i.e., speeding and hard breaking) increased physiological stress and decreased declared trust among drivers, while a safe and lawful automated drive had the opposite effects. Walker et al. (2019) confirmed these physiological observations and showed that a "Perfect" driving style (i.e., smooth braking, stabilized lane keeping) quickly increased trust, but a "Poor" driving style (i.e., hard braking in front of pedestrians, poor lane keeping) slowly decreased it. They also revealed that this trust calibration was very sensitive to recent events as evidenced by a dramatic trust drop when drivers experienced a "Poor" drive after several "Perfect" ones. It should be noted that this effect was observed during a 45-minute single session of video-simulation, with no possible interactions between drivers and $A D$ system, and ultimately no Take-Over Requests (TOR).

TiAD has been mostly evaluated through questionnaires (Manchon et al., 2020). However, several studies also revealed a link between drivers' trust in automation and (a) visual monitoring strategies (Hergeth et al., 2016; Navarro et al., 2019; Walker et al., 2019) and (b) driver's activities (Manchon et al., submitted) during automated driving.

Altogether, automation driving style, either positive or negative, is expected to influence declared trust, on-board driver's activities, visual strategies, and TOR performance. Positive (smooth) driving style is hypothesized to increase declared level of trust and decrease road monitoring while the opposite effects could be expected for negative (abrupt) driving style. A previous experiment showed that the initial level of trust had an influence on early trust calibration, driver's behavior, and road monitoring strategy 
(Manchon et al., submitted). Building up on these results, the present study focuses on trust evolution depending on both initial level of trust, and automation driving style. Trust is expected to be higher among Trustful participants compared with Distrustful participants ( $\mathrm{H} 1)$ and for participants that experienced positive driving style compared with negative driving style $(\mathrm{H} 2)$. An interaction between automation driving style and initial level of trust is also hypothesized. A more drastic decrease of trust is expected for Distrustful drivers who experience a negative driving style as compared to Trustful drivers with that same negative driving style $(\mathrm{H} 3)$.

Additionally, a number of studies demonstrated a positive effect of driver's experience with an automated driving system on TiAD, even in single driving sessions (Gold et al., 2015; Hartwich, Witzlack, et al., 2018; Hergeth et al., 2017; Körber, Baseler, et al., 2018; Kraus, Scholz, Messner, et al., 2020). This effect of experience with automation is thus likely to influence trust and visual strategies even more over several driving sessions. In the present study participants will take part to two driving sessions on different days, trust in automation is expected to increase over time (H4). Nonetheless, only a few studies inquired trust evolution after automation use, during non-utilization period (Hartwich, Beggiato, et al., 2018; Hartwich, Witzlack, et al., 2018; Walker et al., 2018). As TiA evolves both during interaction and non-interaction with automation (Lee \& See, 2004), it seems crucial to address this question. The present experiment aims to better understand how TiAD will evolve during a first interaction (Day 1), either positive or negative, and during a second driving session (Day 2) following a non-utilization period of one week, then during a longer period of non-utilization of four months. An increase in trust is expected after the non-utilization period $(\mathrm{H} 5)$, but trust is expected to remain stable four month after 
the experiment $(\mathrm{H} 6)$. Distrustful drivers are expected to better perform during take-over than Trustful ones (H7). Finally, the unplanned TOR is expected to produce a trust drop among all drivers (H8).

\section{METHOD}

\section{Participants}

Sixty-one healthy drivers $(31 \mathrm{~F} / 30 \mathrm{M}, \mathrm{M}=40.8, \mathrm{SD}=12.4$ years old $)$ participated in this driving simulator study. All participants had a valid driver's license for a minimum of three years $(M=21, S D=12.3)$ and drove regularly. All drivers had normal or corrected-tonormal vision and they received $100 €$ of compensation for their participation. This research complied with the American Psychological Association Code of Ethics and the European law on General Data Protection Regulation. Informed consent was obtained from each participant.

\section{Apparatus}

The study was conducted in a static driving simulator equipped with three $2520 \times 1440 \mathrm{~mm}$ panels, giving a $200^{\circ}$ horizontal field of view. Three 7" 16/9 LCD screens, placed at the same spots the rear mirrors would have been in a car, simulated rear mirrors by displaying a rear view of the driving environment. The dashboard was showed on a 10" 16/9 LCD screen set behind the steering wheel (Figure 1). The SCANeR ${ }^{\text {TM }}$ Studio 1.8 software developed by AV Simulation, France (https://www.avsimulation.fr) was used to run the driving simulation. A 10.1" touch screen was set on the left of the steering wheel as HMI. On the left sideband of the $\mathrm{HMI}$, different pictograms were displayed to indicate the state of the automated driving system (i.e., "Manual driving", "Available HAD”, "Activated HAD", or "Take-over request"), and some specific vehicle maneuvers when relevant (e.g., 
overtaking, braking). The rest of the HMI displayed an Android ${ }^{\mathrm{TM}}$ emulator with some games (e.g., Solitaire, Sudoku, Mahjong) and internet access (with e.g., YouTube ${ }^{\mathrm{TM}}$ and radio app). The eye-tracking system was composed of four $60 \mathrm{~Hz}$ infrared cameras recording data through the Smart-Eye Pro 6.2 software.

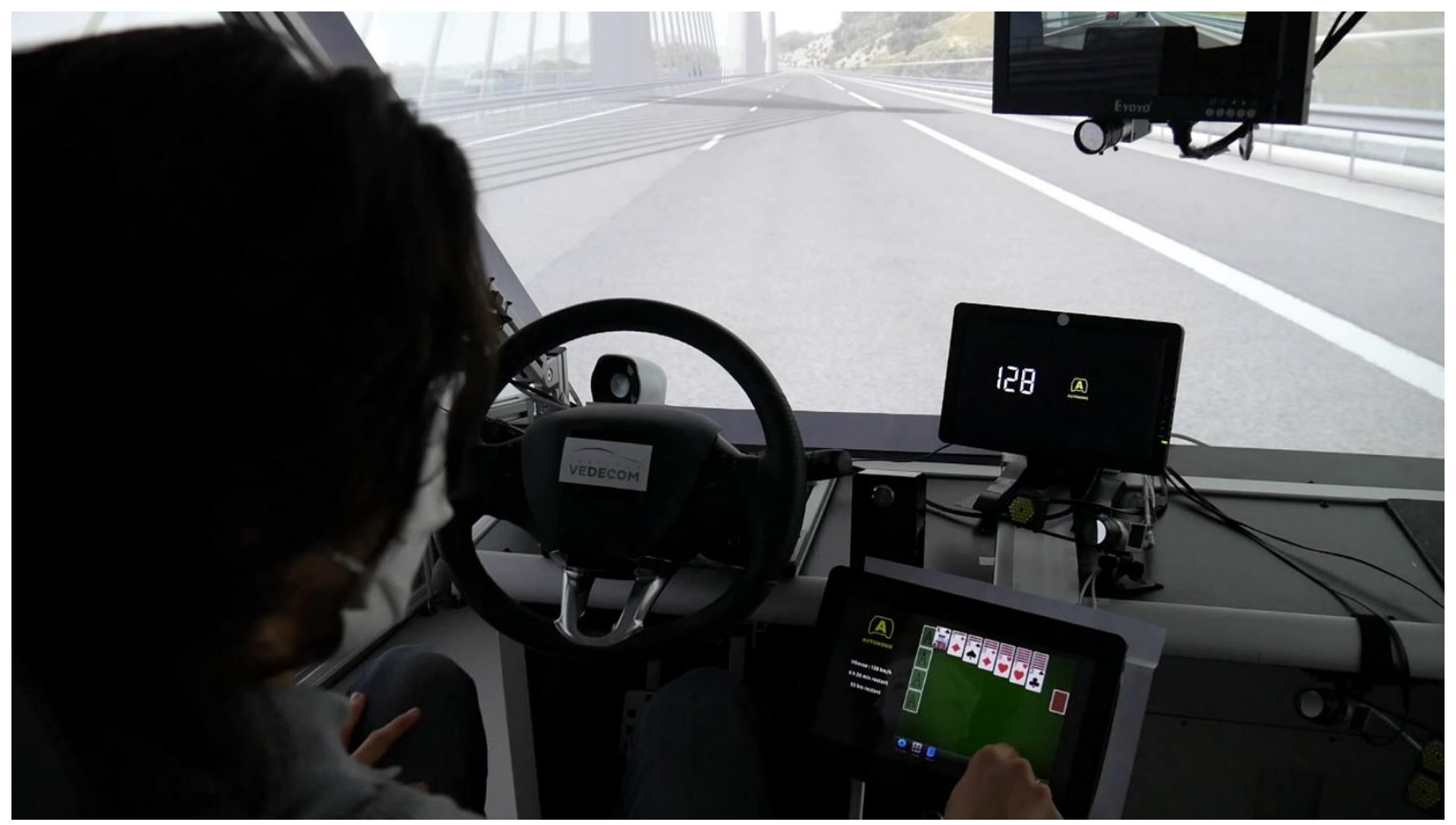

Figure 1. Driving simulator set up.

\section{Level of trust assessment}

Initial level of TiAD was measured using a 10-item questionnaire on a 6-point Likert scale for items 1 to 9 and on a 0 to $100 \%$ rating scale for item 10 (Table 1). These questions were adapted from different trust questionnaires and used in a previous study (Manchon et al., submitted). Following methodology and results from this previous study, trust score served to establish participants' initial level of TiAD, and to create two experimental groups: Trustful drivers (high initial level of trust), were selected for having a trust score over 65\%, and Distrustful drivers (low initial level of trust) were selected for having a trust 
score under $35 \%$. Considering TiAD as normally distributed in the population, centered on 50 with standard deviation of 10 , these thresholds correspond to minus than -1.5 or more than 1.5 standard deviation from the mean $(z \leq-1.5$ or $z \geq 1.5)$. The data showed a pseudo-normal distribution $(\mu=57.8, \sigma=20.5)$, and the thresholds really corresponded to $z \leq-1.1$ or $z \geq .3$. The same scale formulated in past tense was used to assess final level of trust at the end of the first and second day of the experiment.

Table 1. Initial level of TiAD assessment scale

\section{Questions}

1 I would feel safe in an automated vehicle.

O'Cass and Carlson (2012)

2 The automated driving system provides me with more safety compared to Payre et al. (2016) manual driving.

$3^{*}$ I would rather keep manual control of my vehicle than delegate it to the Payre et al. (2016) automated driving system on every occasion.

4 I would trust automated driving system decisions.

O'Cass and Carlson (2012)

5 I would trust automated driving system capacities to manage complex driving situations.

Egea and González (2011)

6 If the weather conditions were bad (e.g., fog, glare, rain), I would delegate the driving task to the automated driving system.

Payre (2015)

7 Rather than monitoring the driving environment, I could focus on other activities confidently.

Egea and González (2011)

8 If driving was boring for me, I would rather delegate it to the automated driving Payre et al. (2016) system than do it myself.

9 I would delegate the driving to the automated driving system if I was tired.

Payre et al. (2016)

10 How much do you trust automated driving systems?

Lee and Moray (1994)

*Answers were inverted for scoring.

Before the experimental session, 165 potential participants were assessed with the initial trust scale by email. Internal consistency and homogeneity (Revelle \& Zinbarg, 2009) 
were good (Cronbach's $\alpha=.93$, McDonald's $\omega_{h}=.67$, and $\omega_{t}=.94$ ). Based on the thresholds presented before, sixty-one participants were selected and divided in two groups: Trustful drivers $\left(n=36,14 F / 22 M, M=41.2, S D=13.3\right.$ years old, $M_{\text {trust }}=78.4$, $\left.S D_{\text {trust }}=9.64\right)$ and Distrustful drivers $(n=25,18 F / 7 M, M=40.1, S D=11.0$ years old, $M_{\text {trust }}=28.3, S_{\text {trust }}=5.99$ ).

The level of trust during automated driving (dynamic trust) was measured at several points of the experiment using a Single Trust Item ranging from 0\% to 100\% ("How much do you trust the automated driving system?", Lee \& Moray, 1994).

\section{Procedure}

The experiment was split in two sessions, separated by approximately a week (min: 4 days, max: $10, M=6.31)$. In the first session, participants signed a consent form, then they received a short description about the upcoming experiment. They were told the highly automated driving (HAD) system was able to manage lateral and longitudinal control in any situation on highway, allowing them to disengage from the environment monitoring task. They were also told they were free to engage in any non-driving related activity (NDRA) they imagined themselves doing during a real automated driving (e.g., reading, texting, using the tablet, listening to the radio). They were showed the specific pictograms corresponding to the HAD states and traffic situations (i.e., lane changing, deceleration, obstacle ahead, and no lane marking) as well as the associated informative sounds. They were finally informed the HAD system could only manage driving on the highway and would trigger a planned TOR if exiting the highway was necessary. A sevenminute simulator practice included HAD activation, a non-critical overtaking maneuver, and a planned TOR before exiting the highway. Participants then started the experimental 
drive in manual mode for few seconds before merging into a two-lane highway and activating HAD.

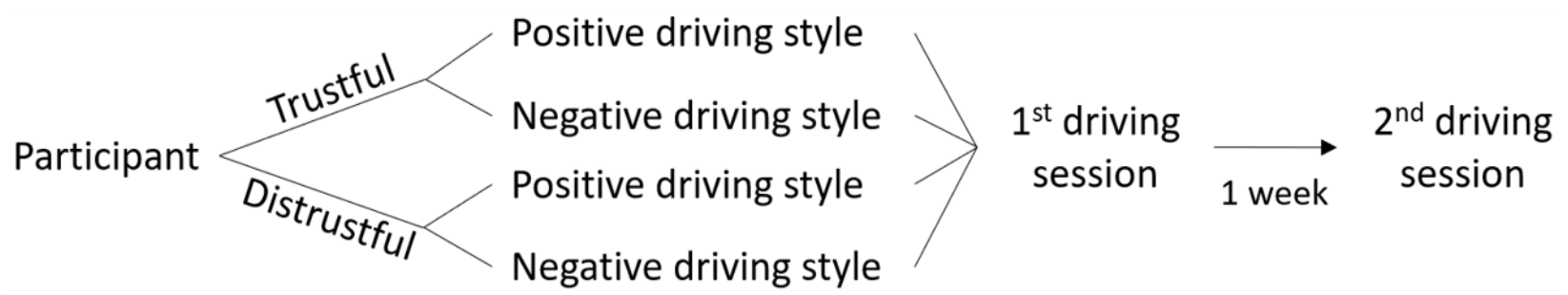

Figure 2. Experimental design.

They were then randomly assigned to the driving style condition (either positive or negative, detailed later) for the rest of the experiment (Figure 2). Driving style was a between-subject variable and participants were not aware of that. The two driving sessions were divided into monotonous driving time $\left(P_{n}\right)$, interspersed with specific situations $\left(S_{n}\right)$. These maneuvers were either managed smoothly or abruptly depending on the assigned driving style condition. Right after each situation but also during monotonous $\mathrm{AD}$, a Single Trust Item ranging from $0 \%$ to $100 \%$ appeared on the $\mathrm{HMI}$ to investigate drivers' dynamic level of trust. During the monotonous driving periods, the ego vehicle drove in the right lane, at a constant speed of $128 \mathrm{~km} / \mathrm{h}$ (the maximum allowed speed on French highways is $130 \mathrm{~km} / \mathrm{h}$ ), while some other vehicles drove around (6-12 vehicles per kilometers) and sometimes overtook the ego vehicle.

Nine situations happened during the two driving sessions. Six were managed by the driving system, two were planned TORs, and the last one was an unplanned TOR. Details about the two different driving styles (positive or negative) during the situations and the TORs are given in Table 2 . 
Table 2. Technical information about situation management and TORs.

\begin{tabular}{|c|c|c|c|c|}
\hline Scenario & & Parameter & $\begin{array}{l}\text { Smooth driving } \\
\text { style condition }\end{array}$ & $\begin{array}{l}\text { Abrupt driving style } \\
\text { condition }\end{array}$ \\
\hline \multirow{3}{*}{ Roadwork area } & \multirow{3}{*}{ A } & TTC before deceleration (s) & 7 & 3 \\
\hline & & Deceleration velocity $\left(\mathrm{m} / \mathrm{s}^{-2}\right)$ & from 128 to 90 & from 128 to 60 \\
\hline & & Lane changing duration (s) & 6 & 3 \\
\hline \multirow{2}{*}{ Slow truck } & & TTC before deceleration (s) & 5 & 3 \\
\hline & & Deceleration velocity $\left(\mathrm{m} / \mathrm{s}^{-2}\right)$ & from 128 to 80 & from 128 to 70 \\
\hline \multirow{2}{*}{ Crossing deer } & & TTC before deceleration (s) & 6 & 3 \\
\hline & & Deceleration velocity $\left(\mathrm{m} / \mathrm{s}^{-2}\right)$ & from 128 to 80 & from 128 to 60 \\
\hline \multirow{2}{*}{$\begin{array}{l}\text { No lane } \\
\text { marking }\end{array}$} & & Deceleration velocity $\left(\mathrm{m} / \mathrm{s}^{-2}\right)$ & from 128 to 110 & from 128 to 120 \\
\hline & & Max lateral lane shift $(\mathrm{m})$ & 0.5 & 1.2 \\
\hline \multirow{3}{*}{$\begin{array}{r}\text { Obstacle } \\
\text { on the road }\end{array}$} & & TTC before deceleration (s) & 4 & 2 \\
\hline & & Deceleration velocity $\left(\mathrm{m} / \mathrm{s}^{-2}\right)$ & from 128 to 100 & from 128 to 100 \\
\hline & & Lane changing duration (s) & 3 & 2 \\
\hline Planned TOR & & Budget time (s) & \multicolumn{2}{|c|}{20} \\
\hline \multirow{2}{*}{$\begin{array}{r}\text { Unplanned } \\
\text { TOR }\end{array}$} & & TTC before TOR (s) & \multicolumn{2}{|c|}{8} \\
\hline & & $\begin{array}{r}\text { TTC before complete } \\
\text { system failure }(\mathrm{s})\end{array}$ & \multicolumn{2}{|c|}{5} \\
\hline
\end{tabular}

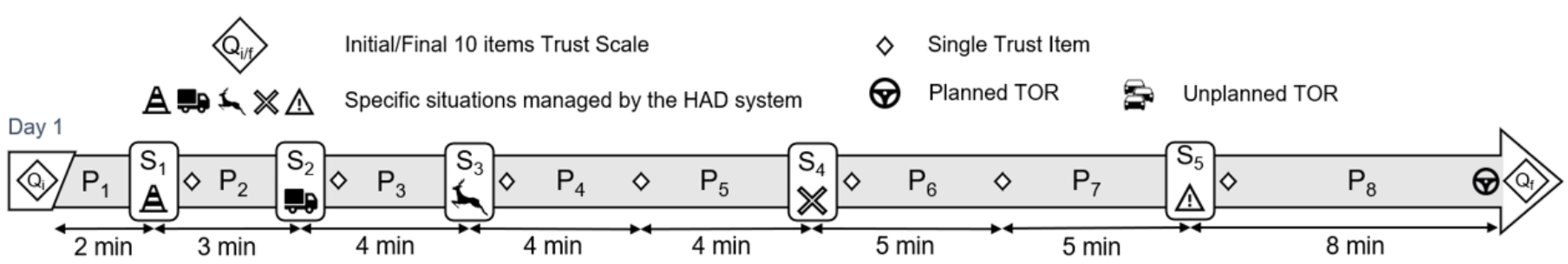

Day 2

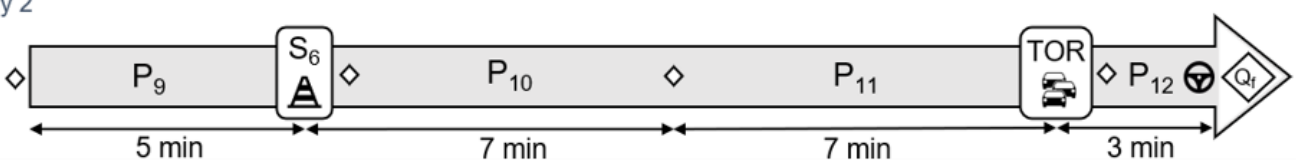

Figure 3. Procedure

During the first driving session, drivers were confronted with five situations in total (Figure

3). The first situation $\left(\mathrm{S}_{1}\right)$ was a roadwork area signaled by traffic cones and a blinking orange arrow on top of a roadwork utility vehicle. The ego vehicle had to decelerate, change from the right to the left lane, then went back to the right lane. In the second situation $\left(\mathrm{S}_{2}\right)$, a slow truck $(80 \mathrm{~km} / \mathrm{h})$ was close to exit highway while the left lane was congested. The ego vehicle had to decelerate until the truck had exited. In the third 
situation $\left(\mathrm{S}_{3}\right)$, a deer suddenly crossing the highway forced the ego vehicle to decelerate. These three first situations were closely related in time in order to reinforce the positive or negative effect of the driving style. In the fourth situation $\left(\mathrm{S}_{4}\right)$, the ego vehicle started to zigzag during a small portion of road with no lane marking. The final situation $\left(\mathrm{S}_{5}\right)$ was represented by an obstacle on the ego lane (a box hidden by the lead vehicle), that forced the ego vehicle to change lane. After eight-minute of monotonous driving, a planned TOR was triggered by a vocal message and orange pictograms, twenty seconds before the highway exit, and drivers were asked to exit the highway and fill the final trust scale. They were debriefed to further investigate their feeling during the driving session, thanked and asked to come back the next week.

The second driving session was shorter and included fewer situations. Participants started on the same track than during the first session, initiated manual driving on the highway and activated HAD with the same characteristics as the previous session. Drivers were firstly confronted with a roadwork area scenario $\left(S_{6}\right.$, with the exact same specifications than the corresponding scenario in the first session, $\mathbf{S}_{1}$ ). After 15 -minute monotonous driving, the last scenario was an unplanned TOR, resulting of a complete system failure in a tunnel, while the ego vehicle approached a traffic jam. The TOR was triggered with a Time To Collision (TTC) of seven seconds, by a continuous and increasingly pressing alarm, with red pictograms on the dashboard and HMI. The system proposed to activate HAD again quickly after the traffic jam was over. The session finished by a three-minute monotonous drive, then a planned TOR was triggered twenty second before the highway exit. They filled a final trust scale again and were debriefed, then rewarded. Four months after, they filled a last trust scale that was sent by email. 


\section{Data analysis}

Dependent variables included declared trust, visual strategies, and TOR performances.

Trust scales were scored by adding each answer, reversing item 3 (cf. Table 1). Scores were then converted in percentage. Single Trust Items were used without transformation.

Visual strategies data (time spent looking towards specific Areas of Interest, $\mathrm{AOI}$, in percentage) were processed following ISO 15007 recommendations. Areas of interest were defined as "Road", "Rear Mirrors", "Dashboard", "HMl”, "Mobile Phone" and "Other" for all glances directed somewhere else. "Road", "Rear Mirrors", and "Dashboard" were grouped in a category "Driving environment" for analysis.

TOR data (including Reaction Time, RT, number of collisions, and TTC profiles) were acquired collecting drivers' inputs through the Acquisition module and captured with the Record module from SCANeR Studio 1.8. Only the unplanned TOR data has been kept.

Data and graphics were processed using R (R Core Team, 2017) and ggplot2 (Wickham, 2016).

\section{RESULTS}

The subsequent analyses follow the mixed-design of the experiment. Between-subject variables were Group: Trustful vs Distrustful and Driving Style: Positive vs Negative. Within-subject variable was Time, with Initial-Day 1, Final-Day 1, Initial-Day 2, and FinalDay 2 for declarative trust using the 10-item questionnaire, with the eleven Single Trust Item measurement points for declarative trust during the drives, or with the twelve time periods between Scenarios ( $P_{1}$ to $P_{12}$, cf Figure 3 ) for drivers' visual strategies.

\section{Trust questionnaires}


A three-way mixed-design ANOVA was performed to investigate the effects of initial level of trust, driving style, and experience with AD on declared trust (Figure 4).

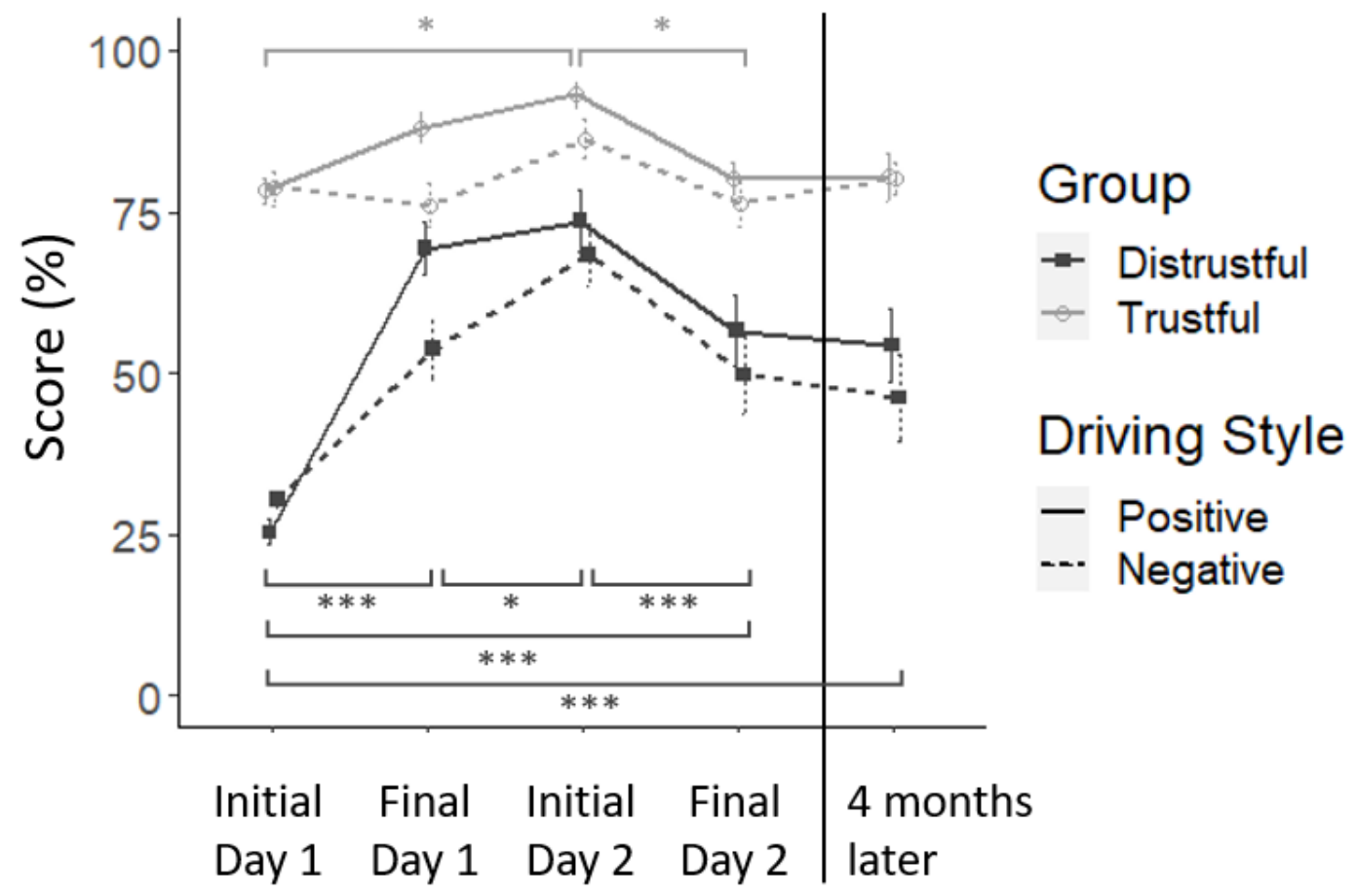

Figure 4. Initial and final trust assessments for the two experimental days.

Error bars: standard error. *: $p<.05 ; * *: p<.01 ; * * *: p<.001$.

There was a significant effect of the Group, $F(1,57)=158.5, p<.001, \eta_{p}^{2}=.735$, and the Time, $F(4,228)=37.1, p<.001, \eta_{p}^{2}=.394$. Post-hoc tests using Bonferroni correction showed that the Initial-Day 1 assessment differed significantly from Final-Day $1(p<.001)$, Final-Day 1 differed from Initial-Day $2(p<.05)$ and Initial-Day 2 differed from Final-Day $2(p<.001)$. In short, declared trust was found to increase during the first session $(p<.05)$, and between the first and second session $(p<.05)$. It then decreased during the second session $(p<.05)$. Trust evolution patterns were different between Trustful and Distrustful participants: Distrustful participants gained trust during the first session $(p<.05)$ while it remained stable for Trustful ones $(p>.1)$. Moreover, trust increased for both groups between the first and the second session $(p<.05)$, during the non-utilization period of 
one week (Figure 4). Trust dropped for both groups (Trustful: $p<.05$ and Distrustful: $p$ $<.001)$ at the end of Day 2 but remained higher than initial trust of Day 1 for Distrustful participants $(p<.001)$ and got back to its initial level for Trustful participant $(p>.1)$. Four months later, trust was still different from Initial-Day $1(p<.001)$ but was not different from Final-Day $2(p>$.1) among Distrustful drivers. Trust was not different from Initial-Day 1 $(p>$.1) nor Final-Day $2(p>$.1) for Trustful ones.

Considering the overall trust evolution during both driving sessions, a repeated measures ANOVA (Greenhouse-Geisser-corrected) was conducted integrating the eleven Single Trust Item measures (Figure 5).

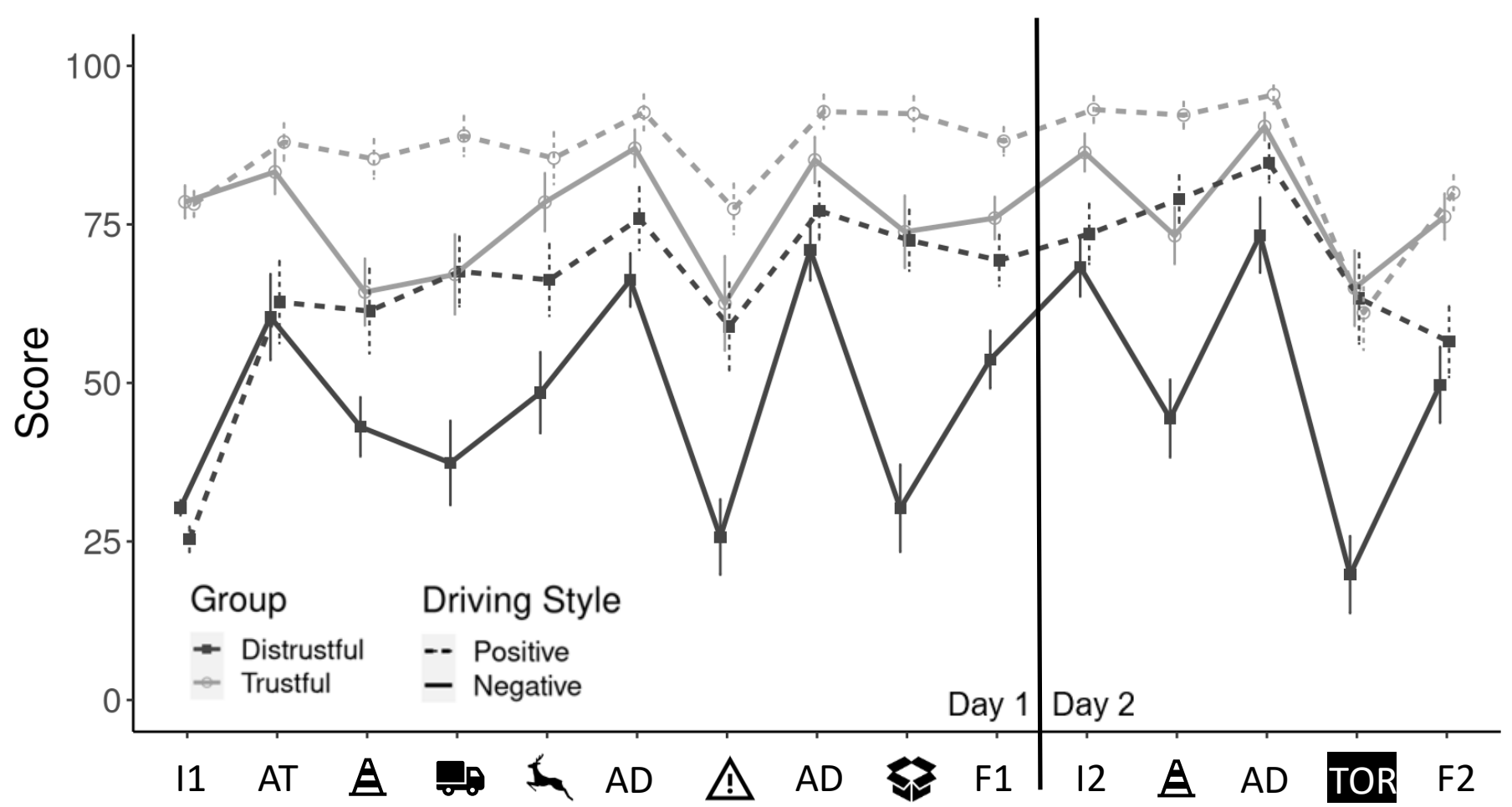

Figure 5. Overall trust evolution during both driving sessions. I: Initial; F: Final; AT: After Training; AD: (monotonous) Automated Driving; TOR: (unplanned) Take-Over Request

There was a significant effect of the Group, $F(1,57)=70.34, p<.001, \eta_{p}^{2}=.552$, the Driving Style, $F(1,57)=23.29, \mathrm{p}<.001, \eta_{\mathrm{p}}^{2}=.290$, and the Time, $F(7.35,418.68)=31.34$, 
$p<.001, \eta_{p}^{2}=.355$, Post-hoc tests using Bonferroni correction showed that declared trust in $S_{6}($ Day 2$)$ was superior than $S_{1}$ (Day 1$)(p>.05)$, while the scenarios were exactly the same (roadwork). Moreover, Time interacted with Group, $F(7.35,418.68)=5.49, p<.001$, $\eta_{p}^{2}=0.088$, indicating a difference in trust calibration depending on the initial level of trust.

Distrustful participants gained significantly more trust than Trustful ones. Time also interacted with Driving Style, $F(7.35,418.68)=7.31, p<.001, \eta_{p}^{2}=.114$, indicating that driving styles induced different trust calibration patterns, more stable for the Positive driving style, more erratic for the Negative one. Finally, a three-way interaction, $F(7.35$, 418.68), $p=.001, \eta_{p}^{2}=.056$, showed a difference in trust evolution over time depending on both the initial level of trust and the driving style. Distrustful participants' trust varied more in the Negative driving style condition, compared to the other combinations.

\section{Visual behavior}

Due to recording problems, 4 participants were excluded from the visual behavior analysis. For the remaining 57 drivers, visual strategies towards the driving environment were decomposed in Periods $\left(\mathrm{P}_{1}\right.$ to $\left.\mathrm{P}_{12}\right)$, corresponding to the time lapse of monotonous automated driving between specific scenarios and trust measurement points. Periods of time drivers were looking towards the driving environment were compared: a three-way mixed-design ANOVA (Group $x$ Driving Style $x$ Periods (12 levels: $P_{1}$ to $\left.P_{12}\right)$ ) was conducted to investigate the evolution of driving environment monitoring during the drives (Figure 6).

The effect of the Group, $F(1,53)=35.04, p<.001, \eta_{p}^{2}=.398$, indicated that Distrustful participants monitored significantly more the driving environment than Trustful ones. No effect of the Driving Style was found $(p>.1)$. The effect of the Periods, $F(11,583)=6.44$, 
$p<.001, \eta_{p}^{2}=.108$, showed a decrease in driving environment monitoring over time,

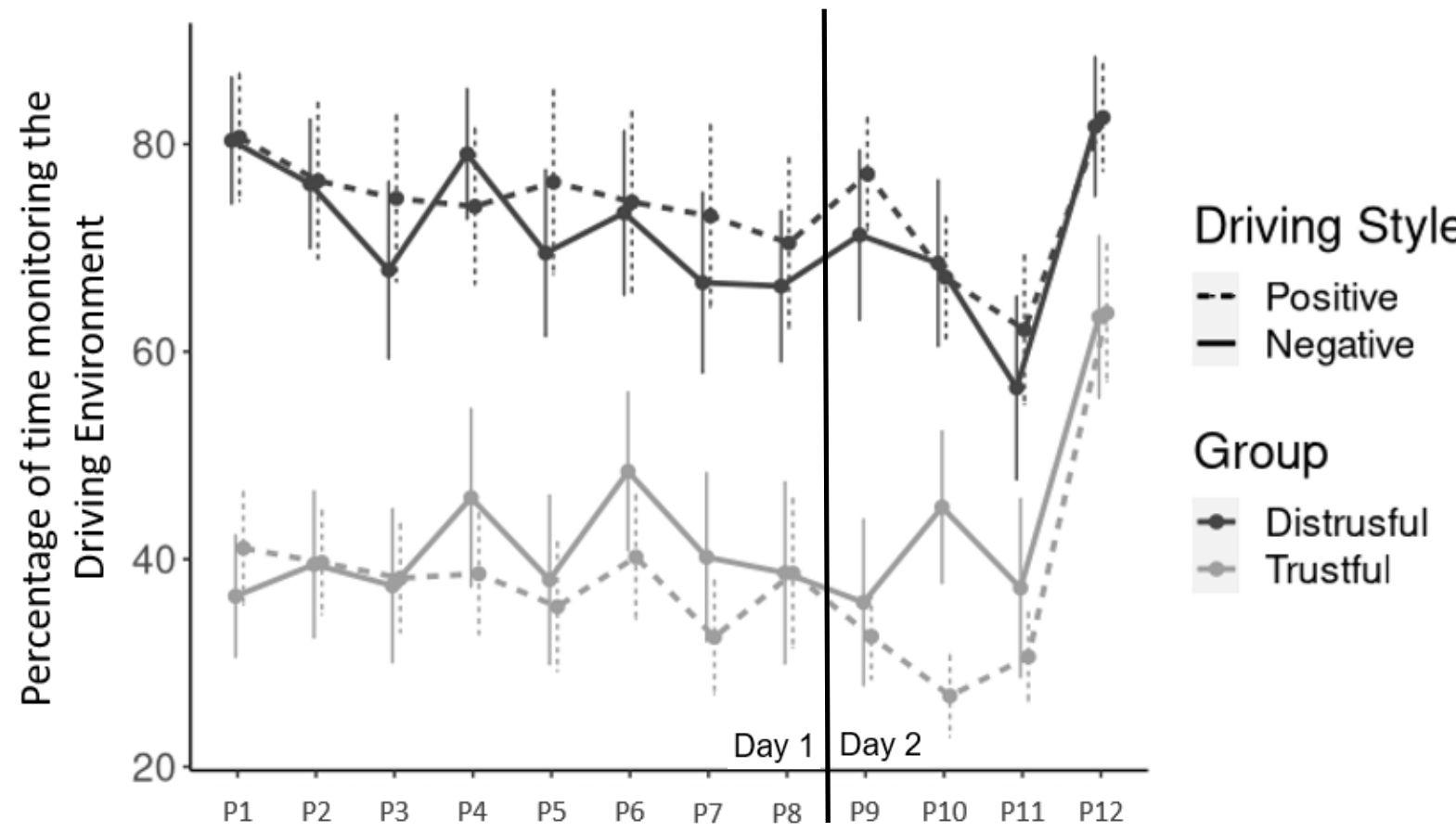

Figure 6. Drivers' monitoring of the driving environment during the monotonous Automated Driving periods for both drives

except for the last five minutes following the unplanned TOR, when the road monitoring increased significantly. Post-hoc tests using Bonferroni correction showed this progression was slow and linear, with no particular breakpoint. No interaction effects were found $(p>.1)$.

To examine drivers' visual strategies during specific scenarios, a three-way repeated measures ANOVA (Group x Driving Style $x$ Scenario ( 7 levels: 6 scenarios and the TOR)) was conducted (Figure 7). Results indicated an effect of the Group, $F(1,53)=26.57, p$ $\left.<.001, \eta_{p}^{2}=.326,\right)$. An effect of the Scenario, $F(6,318)=4.33, p<.001, \eta_{p}^{2}=.073$ showed different visual strategies depending on the scenario. Post-hoc tests using Bonferroni correction pointed out the TOR scenario was different from the other scenarios, except for $S_{4}$ (No marking). An interaction Group x Scenario, $F(6,318)=2.42, p<.05$, 
$\eta_{p}^{2}=.042$ indicated the evolution of visual behavior during scenarios differed depending on drivers' initial level of trust. Distrustful drivers monitored more the driving environment and in a more stable way among scenarios, while Trustful drivers had significant differences in environment monitoring among scenarios.

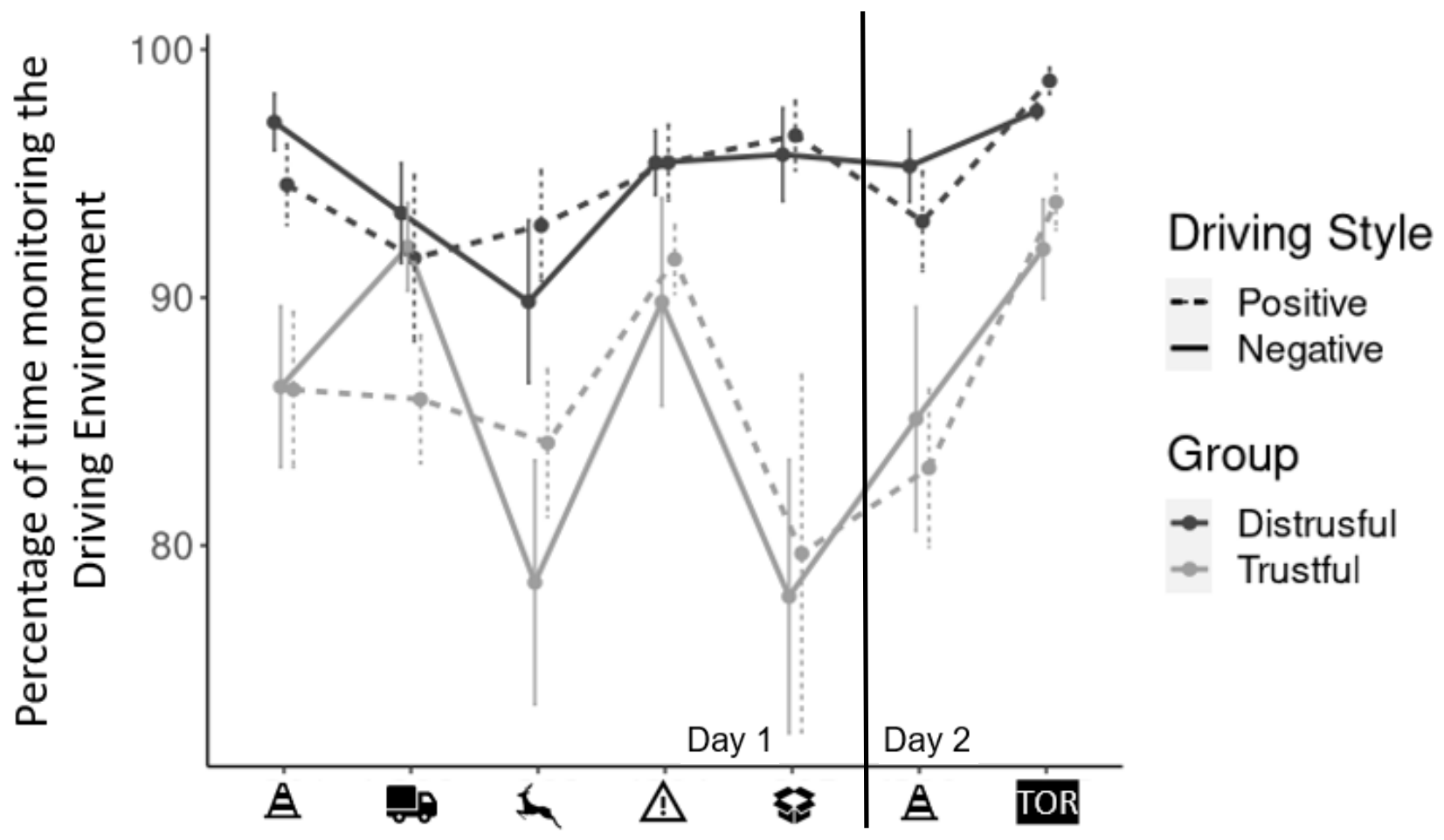

Figure 7. Drivers' monitoring of the driving environment during the specific scenarios for both drives

\section{TOR performances}

TOR performances were inquired examining RT, number of collisions, and TTC. Planned TORs data were not analyzed here, as the focus was set on the unplanned TOR.

No difference was showed in RT depending on the initial level of trust, nor the driving style (Table 3). Thirty three percent (20/61) of the participants collided with the stopped car in front of the ego vehicle, or with the highway side barrier during the TOR situation. 
Table 3. RT (s) and collisions number during the unplanned TOR.

\begin{tabular}{|c|c|c|c|c|c|c|c|c|}
\hline Group & Driving Style & $\begin{array}{r}\text { Minimum } \\
\text { RT }\end{array}$ & $\begin{array}{r}1 s t \\
\text { quartile }\end{array}$ & Median & $\begin{array}{r}\text { Mean } \\
\text { RT }\end{array}$ & $\begin{array}{r}\text { 3rd } \\
\text { quartile }\end{array}$ & $\begin{array}{r}\text { Maximum } \\
\text { RT }\end{array}$ & $\begin{array}{r}\text { Collisions } \\
\text { number }\end{array}$ \\
\hline \multirow{2}{*}{ Distrustful } & Positive & 2.45 & 3.46 & 3.78 & 4.12 & 5.39 & 6.00 & 4 \\
\hline & Negative & 2.15 & 2.75 & 3.90 & 3.98 & 5.25 & 5.90 & 6 \\
\hline \multirow{2}{*}{ Trustful } & Positive & 2.65 & 3.28 & 3.68 & 3.95 & 4.19 & 5.95 & 4 \\
\hline & Negative & 2.80 & 3.48 & 4.05 & 4.05 & 4.25 & 5.99 & 6 \\
\hline
\end{tabular}

TTC profiles are presented in Figure 8. A repeated measure ANOVA showed no main differences in Group nor Driving Style, but revealed an interaction between Group and Time, $F(16,576), p=.05, \eta_{p}^{2}=.044$. This indicated a difference of TTC variation depending on the initial level of trust: Distrustful participants braked harder than Trustful ones.

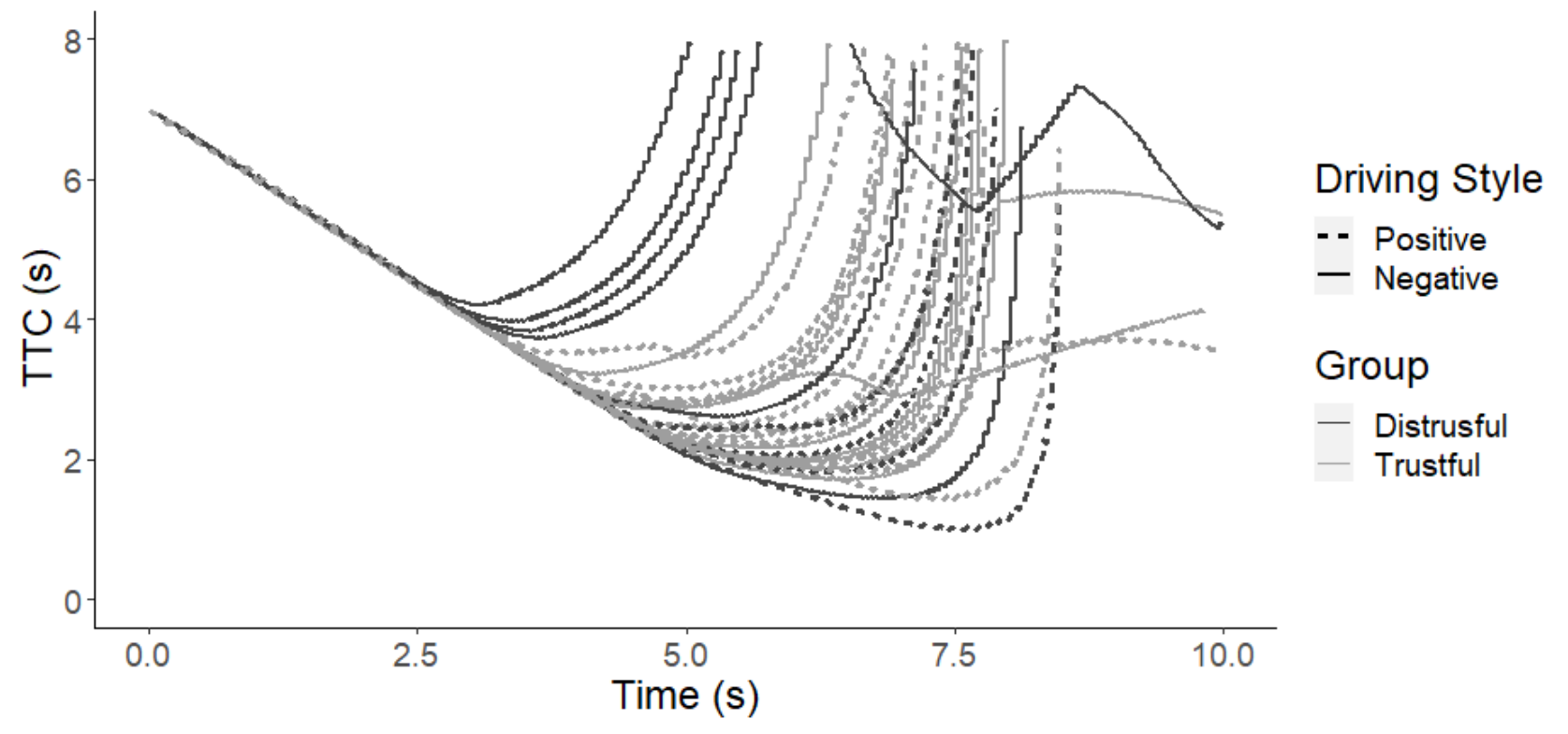

Figure 8. TTC profile for both groups

\section{Discussion}

The present study aimed to investigate the effect of an either high or low initial level of TiAD (Trustful or Distrustful), on driver's trust construction and evolution during automated driving. It also aimed to inquire the effect of a longer experience with an automated driving system, including two driving sessions separated by a week of non-utilization, then a last 
trust measurement four months after, on drivers' TiAD evolution. Another goal was to assess the possibility to influence trust calibration with an either positive or negative HAD's driving style. Finally, the study aimed to investigate the effect of these factors on TOR performance under time constraint.

\section{Effect of the initial level trust}

Declared trust results showed a persistent difference between Trustful and Distrustful participants. Despite both groups' trust increase, this initial difference in trust remained all along the experiment duration, confirming $\mathrm{H} 1$. This result suggests that initial learned trust have an enduring influence on trust, even after the first interaction with a HAD system. That being said, the trust progression among Distrustful drivers indicates that several positive interactions may be enough to build a high level of TiAD. The increase in trust is coherent with previous study (e.g., Bueno et al., 2016; Gold et al., 2015; Körber et al., 2018; Kraus et al., 2020).

Differences in declared trust were also confirmed when examining the drivers' visual behavior. Distrustful participants monitored the driving environment twice more than Trustful ones during the monotonous HAD, corroborating $\mathrm{H} 1$. This result is coherent with previous findings (Hergeth et al., 2016; Manchon et al., submitted; Payre et al., 2017; Schwarz et al., 2019) and shows how the initial level of trust may influence drivers' visual behavior.

Surprisingly, no difference depending on the initial level of trust was found on RT during the TOR, conflicting with results from Körber et al. (2018) and H7. This may be due to the significant trust increase occurring during the experiment, that led both groups to over- 
estimate the HAD system capabilities. However, TTC patterns were different during this event: Distrustful drivers braked harder than Trustful ones. This might result of a higher level of stress during the take-over. It supports again the importance of assessing the initial level of trust in future studies, as it seems to be a determinant of drivers' behavior during HAD.

\section{Effect of the driving style}

HAD driving style appeared to be able to influence declared trust, in accordance with previous studies (Hartwich et al., 2020; Schmidt et al., 2021; Walker et al., 2019) and H2. It also seemed to interact with the initial level of trust, showing different trust evolution pattern depending on both the initial level and the driving style, and supporting $\mathrm{H} 3$. Nevertheless, no main effect of the driving style has been found on visual strategies nor TOR performances. This may be due to visual control reflexes while driving, that had more influence on participants behavior than HAD driving style. This suggests that visual strategies may not always be a robust measurement of TiAD and should be considered along with other data. It may also be linked to the decrease in perceived risks induced by the driving simulator, compared to a real driving, that might have pushed participants to monitor less the driving environment. The mismatch between declared trust and measured behavior may also confirm that factors linked to dynamic trust have less influence on driver's behavior than factors linked to initial learned trust, as suggested by Hoff and Bashir (2015). Declared trust and behavioral trust might be divergent in some situations, such as potentially harmful driving situations.

\section{Effect of the time and experience}


Declared trust results showed a significant increase in trust during the early interaction with the system (supporting $\mathrm{H} 4$ ), particularly during the first five-minute of the first driving session, and particularly for Distrustful participants. Trust evolution patterns were different among Trustful and Distrustful participants. This may indicate a mismatch between their initial mental representation and their actual perceptions during simulated HAD. After the initial trust increase, trust grew slowly (in line with Hartwich et al., 2018; Hergeth et al., 2016; Körber et al., 2018), and decreased punctually in case of unexpected events during HAD (Hergeth et al., 2015; Kraus, Scholz, Stiegemeier, et al., 2020). It varied widely during the drives, particularly among Distrustful participants, confirming H3. This evolution pattern may indicate Distrustful participants are more sensitive than Trustful ones and are more affected by HAD negative driving style. After the experiment, trust seemed to remain stable for both groups, confirming $\mathrm{H} 6$ and suggesting that even a relatively short experience with a HAD system can lead to persistent evolution in TiAD, at least for Distrustful drivers.

Drivers' glances towards the Driving Environment decreased gradually during both driving sessions until the unplanned TOR, confirming observation by Schwarz et al. (2019). This confirmed the increase in trust showed by the questionnaires and supports $\mathrm{H} 4$ : behavioral trust may vary distinctly depending on the automated driving performance. Moreover, it is worth mentioning that reaction times were high compared to what was reported in other studies (B. Zhang et al., 2019), and the number of collision was also surprisingly high. This may imply the 65 minutes of experience with the system during the two driving sessions were able to decrease drivers' ability to take manual control back, particularly the one third of the participants who collided with the traffic during the manual take-over. 
High level of TiAD has been shown to correlate with drowsiness (Kundinger et al., 2019) and participants of the current experiment might have experienced the same. This may participate to explain the high number of poor manual take-over. This high level of trust may also have appeared because drivers observed the HAD system performed without incident on several occasions and did not understand why the last situation needed a manual intervention. In any case, trust dropped after the unplanned TOR for both groups, confirming $\mathrm{H} 8$. As hypothesized, the one-week period of non-utilization induced a trust grow among both groups, supporting H5. Moreover, experience with HAD seemed to modify drivers' expectations and related trust. The same scenario $\left(S_{1} / S_{6}\right.$, the roadwork area) inspired more trust the second time, during Day 2, than the first time, during Day 1 , regardless of the HAD driving style. This result supports the idea drivers' expectation and mental model are updated during and after experiencing HAD, and this variation impacts trust (Beggiato et al., 2015; Beggiato \& Krems, 2013; Blömacher et al., 2018, 2020).

\section{CONCLUSION}

According to Hoff and Bashir (2015)'s model, trust may be decomposed in layers. The present study confirms the existence of a dynamic and highly variable TiAD that evolves during the interaction, alongside a more stable TiAD that evolves outside interaction periods.

The initial level of trust was found to deeply impact TiAD calibration during and after HAD in this study. This initial level had a constant influence on declared and behavioral trust and supports the idea that Hoff and Bashir's initial learned trust has a stronger influence than dynamic learned trust during the early stages of interaction with the system. HAD driving style also showed an impact on declared trust, but its effect on behavioral trust 
was unclear. Results confirmed the positive effect of experience on TiAD, and suggested a positive experience is able to induce a state of over-trust among some drivers. Other studies are needed to better understand the calibration of trust on longer periods of time, and to assess the way driver's mental representation of HAD influences the TiAD calibration during these critical first interactions. 


\section{REFERENCES}

Beggiato, M., \& Krems, J. F. (2013). The evolution of mental model, trust and acceptance of adaptive cruise control in relation to initial information. Transportation Research Part F: Traffic Psychology and Behaviour, 18, 47-57. https://doi.org/10.1016/j.trf.2012.12.006

Beggiato, M., Pereira, M., Petzoldt, T., \& Krems, J. (2015). Learning and development of trust, acceptance and the mental model of ACC. A longitudinal on-road study. Transportation Research Part F: Traffic Psychology and Behaviour, 35, 75-84. https://doi.org/10.1016/j.trf.2015.10.005

Beller, J., Heesen, M., \& Vollrath, M. (2013). Improving the Driver-Automation Interaction: An Approach Using Automation Uncertainty. Human Factors: The Journal of the Human Factors and Ergonomics Society, 55(6), 1130-1141. https://doi.org/10.1177/0018720813482327

Blömacher, K., Nöcker, G., \& Huff, M. (2018). The role of system description for conditionally automated vehicles. Transportation Research Part F: Traffic Psychology and Behaviour, 54, 159-170. https://doi.org/10.1016/j.trf.2018.01.010

Blömacher, K., Nöcker, G., \& Huff, M. (2020). The evolution of mental models in relation to initial information while driving automated. Transportation Research Part F: Traffic Psychology and Behaviour, 68, 198-217. https://doi.org/10.1016/j.trf.2019.11.003

Bueno, M., Dogan, E., Selem, F. H., Monacelli, E., Boverie, S., \& Guillaume, A. (2016). How different mental workload levels affect the take-over control after automated driving. Intelligent Transportation Systems (ITSC), 2016 IEEE 19th International Conference On, 2040-2045. 
Feldhütter, A., Gold, C., Hüger, A., \& Bengler, K. (2016). Trust in Automation as a Matter of Media Influence and Experience of Automated Vehicles. Proceedings of the Human Factors and Ergonomics Society Annual Meeting, 60(1), 2024-2028. https://doi.org/10.1177/1541931213601460

Ghazizadeh, M., Lee, J. D., \& Boyle, L. N. (2012). Extending the Technology Acceptance Model to assess automation. Cognition, Technology \& Work, 14(1), 39-49. http://dx.doi.org.bibelec.univ-lyon2.fr/10.1007/s10111-011-0194-3

Gold, C., Körber, M., Hohenberger, C., Lechner, D., \& Bengler, K. (2015). Trust in Automation Before and After the Experience of Take-over Scenarios in a Highly Automated Vehicle. Procedia Manufacturing, 3, 3025-3032. https://doi.org/10.1016/j.promfg.2015.07.847

Hartwich, F., Beggiato, M., \& Krems, J. F. (2018). Driving comfort, enjoyment and acceptance of automated driving - effects of drivers' age and driving style familiarity. Ergonomics, 61(8), 1017-1032. https://doi.org/10.1080/00140139.2018.1441448

Hartwich, F., Schmidt, C., Gräfing, D., \& Krems, J. F. (2020). In the Passenger Seat: Differences in the Perception of Human vs. Automated Vehicle Control and Resulting HMI Demands of Users. In H. Krömker (Ed.), HCI in Mobility, Transport, and Automotive Systems. Automated Driving and In-Vehicle Experience Design (Vol. 12212, pp. 31-45). Springer International Publishing. https://doi.org/10.1007/978-3-030-50523-3_3

Hartwich, F., Witzlack, C., Beggiato, M., \& Krems, J. F. (2018). The first impression counts - A combined driving simulator and test track study on the development of trust and acceptance of highly automated driving. Transportation Research Part F: Traffic Psychology and Behaviour. https://doi.org/10.1016/j.trf.2018.05.012 
Häuslschmid, R., von Bülow, M., Pfleging, B., \& Butz, A. (2017). SupportingTrust in Autonomous Driving. Proceedings of the 22nd International Conference on Intelligent User Interfaces - IUI ’17, 319-329. https://doi.org/10.1145/3025171.3025198

Hergeth, S., Lorenz, L., \& Krems, J. F. (2017). Prior familiarization with takeover requests affects drivers' takeover performance and automation trust. Human Factors, 59(3), 457-470. https://doi.org/10.1177/0018720816678714

Hergeth, S., Lorenz, L., Krems, J., \& Toenert, L. (2015, June 22). Effects of Take-Over Requests and Cultural Background on Automation Trust in Highly Automated Driving. https://doi.org/10.17077/drivingassessment.1591

Hergeth, S., Lorenz, L., \& Vilimek, R. (2016). Keep Your Scanners Peeled: Gaze Behavior as a Measure of Automation Trust During Highly Automated Driving. Human Factors, 3(58), 509-519. https://doi.org/10.1177/0018720815625744

Hoff, K. A., \& Bashir, M. (2015). Trust in automation: Integrating empirical evidence on factors that influence trust. Human Factors, 57(3), 407-434. https://doi.org/10.1177/0018720814547570

Khastgir, S., Birrell, S., Dhadyalla, G., \& Jennings, P. (2018). Calibrating trust through knowledge: Introducing the concept of informed safety for automation in vehicles. Transportation Research Part C: Emerging Technologies, 96, 290-303. https://doi.org/10.1016/j.trc.2018.07.001

Koo, J., Kwac, J., Ju, W., Steinert, M., Leifer, L., \& Nass, C. (2015). Why did my car just do that? Explaining semi-autonomous driving actions to improve driver understanding, trust, and 
performance. International Journal on Interactive Design and Manufacturing (IJIDeM), 9(4), 269-275. https://doi.org/10.1007/s12008-014-0227-2

Körber, M., Baseler, E., \& Bengler, K. (2018). Introduction matters: Manipulating trust in automation and reliance in automated driving. Applied Ergonomics, 66, 18-31. https://doi.org/10.1016/j.apergo.2017.07.006

Körber, M., Prasch, L., \& Bengler, K. (2018). Why Do I Have to Drive Now? Post Hoc Explanations of Takeover Requests. Human Factors, 3(60), 305-323.

Kraus, J., Scholz, D., Messner, E.-M., Messner, M., \& Baumann, M. (2020). Scared to Trust? Predicting Trust in Highly Automated Driving by Depressiveness, Negative SelfEvaluations and State Anxiety. Frontiers in Psychology, 10. https://doi.org/10.3389/fpsyg.2019.02917

Kraus, J., Scholz, D., Stiegemeier, D., \& Baumann, M. (2020). The More You Know: Trust Dynamics and Calibration in Highly Automated Driving and the Effects of Take-Overs, System Malfunction, and System Transparency. Human Factors: The Journal of the Human Factors and Ergonomics Society, 62(5), 718-736. https://doi.org/10.1177/0018720819853686

Kundinger, T., Wintersberger, P., \& Riener, A. (2019). (Over)Trust in Automated Driving: The Sleeping Pill of Tomorrow? Extended Abstracts of the 2019 CHI Conference on Human Factors in Computing Systems, 1-6. https://doi.org/10.1145/3290607.3312869

Lee, J. D., \& Moray, N. (1994). Trust, self-confidence, and operators' adaptation to automation. International Journal of Human-Computer Studies, 40(1), 153-184. https://doi.org/10.1006/ijhc.1994.1007 
Lee, J. D., \& See, K. (2004). Trust in Automation: Designing for Appropriate Reliance. Human Factors, 46(1), 50-80. https://doi.org/10.1518/hfes.46.1.50_30392

Lu, Z., Zhang, B., Feldhütter, A., Happee, R., Martens, M., \& De Winter, J. C. F. (2019). Beyond mere take-over requests: The effects of monitoring requests on driver attention, take-over performance, and acceptance. Transportation Research Part F: Traffic Psychology and Behaviour, 63, 22-37. https://doi.org/10.1016/j.trf.2019.03.018

Manchon, J. B., Bueno, M., \& Navarro, J. (submitted). How the initial level of Trust in Automated Driving impact drivers’ behavior and early trust construction. Manuscript in Submitted for Publication.

Manchon, J. B., Bueno, M., \& Navarro, J. (2020). From manual to automated driving: How does trust evolve? Theoretical Issues in Ergonomics Science, 1-27. https://doi.org/10.1080/1463922X.2020.1830450

Miller, D., Johns, M., Mok, B., Gowda, N., Sirkin, D., Lee, K., \& Ju, W. (2016). Behavioral Measurement of Trust in Automation: The Trust Fall. Proceedings of the Human Factors and Ergonomics Society Annual Meeting, 60(1), 1849-1853. https://doi.org/10.1177/1541931213601422

Morris, D. M., Erno, J. M., \& Pilcher, J. J. (2017). Electrodermal Response and Automation Trust during Simulated Self-Driving Car Use. Proceedings of the Human Factors and Ergonomics Society Annual Meeting, 61(1), 1759-1762. https://doi.org/10.1177/1541931213601921

Navarro, J. (2019). A state of science on highly automated driving. Theoretical Issues in Ergonomics Science, 20(3), 366-396. https://doi.org/10.1080/1463922X.2018.1439544 
Navarro, J., Osiurak, F., Ovigue, M., Charrier, L., \& Reynaud, E. (2019). Highly Automated Driving Impact on Drivers’ Gaze Behaviors during a Car-Following Task. International Journal of Human-Computer Interaction, 1-10. https://doi.org/10.1080/10447318.2018.1561788

Parasuraman, R., \& Riley, V. (1997). Humans and Automation: Use, Misuse, Disuse, Abuse. Human Factors, 39(2), 230-253. https://doi.org/10.1518/001872097778543886

Payre, W., Cestac, J., Dang, N.-T., Vienne, F., \& Delhomme, P. (2017). Impact of training and invehicle task performance on manual control recovery in an automated car. Transportation Research Part F: Traffic Psychology and Behaviour, 46, 216-227. https://doi.org/10.1016/j.trf.2017.02.001

Payre, W., Cestac, J., \& Delhomme, P. (2016). Fully Automated Driving: Impact of Trust and Practice on Manual Control Recovery. Human Factors, 58(2), 229-241. https://doi.org/10.1177/0018720815612319

Revelle, W., \& Zinbarg, R. E. (2009). Coefficients Alpha, Beta, Omega, and the glb: Comments on Sijtsma. Psychometrika, 74(1), 145-154. https://doi.org/10.1007/s11336-008-9102-z

Schmidt, C., Hartwich, F., \& Krems, J. F. (2021). Looking at Driving Automation from a Passenger’s Perspective: Driving Experience and Gaze Behavior During Fully Automated vs. Human Vehicle Control. In T. Ahram, R. Taiar, K. Langlois, \& A. Choplin (Eds.), Human Interaction, Emerging Technologies and Future Applications III (Vol. 1253, pp. 39). Springer International Publishing. https://doi.org/10.1007/978-3-030-55307-4_1 
Schwarz, C., Gaspar, J., \& Brown, T. (2019). The effect of reliability on drivers' trust and behavior in conditional automation. Cognition, Technology \& Work, 21(1), 41-54. https://doi.org/10.1007/s10111-018-0522-y

Walker, F., Boelhouwer, A., Alkim, T., Verwey, W. B., \& Martens, M. H. (2018). Changes in Trust after Driving Level 2 Automated Cars. Journal of Advanced Transportation, 2018, 1-9. https://doi.org/10.1155/2018/1045186

Walker, F., Wang, J., Martens, M. H., \& Verwey, W. B. (2019). Gaze behaviour and electrodermal activity: Objective measures of drivers' trust in automated vehicles. Transportation Research Part F: Traffic Psychology and Behaviour, 64, 401-412. https://doi.org/10.1016/j.trf.2019.05.021

Wintersberger, P., von Sawitzky, T., Frison, A.-K., \& Riener, A. (2017). Traffic Augmentation as a Means to Increase Trust in Automated Driving Systems. Proceedings of the 12th Biannual Conference on Italian SIGCHI Chapter - CHItaly '17, 1-7. https://doi.org/10.1145/3125571.3125600

Zhang, B., de Winter, J., Varotto, S., Happee, R., \& Martens, M. (2019). Determinants of take-over time from automated driving: A meta-analysis of 129 studies. Transportation Research Part F: Traffic Psychology and Behaviour, 64, 285-307. https://doi.org/10.1016/j.trf.2019.04.020

Zhang, T., Tao, D., Qu, X., Zhang, X., Lin, R., \& Zhang, W. (2019). The roles of initial trust and perceived risk in public's acceptance of automated vehicles. Transportation Research Part C: Emerging Technologies, 98, 207-220. https://doi.org/10.1016/j.trc.2018.11.018 